\title{
ROTATING A MEMS INERTIAL MEASUREMENT UNIT FOR A FOOT-MOUNTED PEDESTRIAN NAVIGATION
}

\author{
${ }^{1}$ Khairi Abdulrahim, ${ }^{2}$ Chris Hide, ${ }^{2}$ Terry Moore, ${ }^{2}$ Chris Hill \\ ${ }^{1}$ Faculty of Science and Technology, Universiti Sains Islam Malaysia (USIM), 71800, Nilai, Negeri Sembilan, Malaysia \\ ${ }^{2}$ Nottingham Geospatial Institute, University of Nottingham, UK
}

Received 2014-09-03; Revised 2014-12-17; Accepted 2014-12-30

\begin{abstract}
Pedestrian navigation especially indoors suffers from the unavailability of useful GNSS signals for positioning. Alternatively, a low-cost Inertial Measurement Unit (IMU) positioning system that does not depend on the GNSS signal can be used for indoor navigation. However its performance is still compromised because of the fastaccumulating heading drift error affecting such a low-cost IMU sensor. This results in a huge positioning error when navigating more than a few seconds using only the low-cost sensor. In this study, real field trials results are presented when a foot-mounted IMU is rotated on a single axis. Two promising results have been obtained. First, it mitigates the heading drift error significantly and second, it increases the observability of IMU z-axis gyro bias error. This has resulted in a greatly reduced error in position for the low-cost pedestrian navigation system.
\end{abstract}

Keywords: Heading Drift, IMU, Pedestrian Navigation

\section{INTRODUCTION}

In indoor pedestrian navigation where a good and reliable Global Navigation Satellite System (GNSS) signal is often unavailable, the use of inertial sensors for a navigation system is not uncommon, for example in (Alvarez et al., 2012). Low-cost inertial sensors are more attractive because it is much cheaper in price compared to other technologies such as WIFI-based, map-based, vision-based and magnetic-based navigation technology. Unlike GNSS, it does not require external signals for positioning and navigation. It uses the concept of dead reckoning where the consecutive position and attitude relative to its initial position and initial attitude are computed from the IMU acceleration and gyro data.

However, the use of such a low cost sensor for navigation comes with a cost. Because of the concept of dead reckoning that it uses and because it is also an autonomous sensor, it accumulates error over time. Nonetheless, for a foot-mounted low-cost Pedestrian Navigation System (PNS), the frequent use of Zero Velocity Updates (ZUPT) measurements consistently bounds many of the IMU errors (Foxlin, 2005). However heading drift still remains despite the use of ZUPT because it is not possible to estimate the heading error using only ZUPT measurements.

The focus of this study is therefore on how to address the heading drift problem-caused by the accumulated IMU heading error-which will degrade the accuracy of the IMU position solution (Jiménez et al., 2012). Therefore, in this study, real field trial results are presented to demonstrate an approach to mitigate heading drift for the low-cost footmounted IMU after mechanically rotating the IMU.

This study is presented as follows. Section II gives the mathematical equations describing the Inertial Navigation System (INS) output after undergoing rotations because of the Rotating IMU (RIMU). The results from real field trials are presented in Section III and then Section IV concludes the paper.

\section{THE EFFECT OF SINGLE-AXIS ROTATION OF THE IMU}

\subsection{Modulation of Error}

Velocity error states, $\delta \dot{v}^{n}$ and attitude error states, $\dot{\epsilon}$ can be propagated using a standard strap down Corresponding Author:Khairi Abdulrahim, Faculty of Science and Technology, Universiti Sains Islam Malaysia (USIM), 71800, Nilai, Negeri Sembilan, Malaysia 
error navigation equation with phi-angle error model (Klein et al., 2012) Equation 1 and 2:

$$
\begin{aligned}
& \delta \dot{v}^{n}=C_{b}^{n} \delta f^{b}+C_{b}^{n} f^{b} \times \in-\left(2 \omega_{i e}^{n}+\omega_{e n}^{n}\right) \\
& \times \delta v^{n}-\left(2 \delta \omega_{i e}^{n}+\delta \omega_{e n}^{n}\right) \times v^{n}+\delta g^{n} \\
& \dot{\epsilon}=-\omega_{i n}^{n} \times \in+\delta \omega_{i n}^{n}-C_{b}^{n} \delta \omega_{i b}^{b}
\end{aligned}
$$

In the equations above, $\delta \omega_{i b}^{b}$ and $\delta f^{b}$ are errors caused by the gyroscope and accelerometer sensor errors.

The matrix $C_{b}^{n}$ transforms body frame to navigation frame of an IMU to produce meaningful position solution. When the IMU is rotated about its y-axis, $C_{b}^{n}$ is made to change continuously. This causes the IMU error terms for the $\mathrm{x}$-axis and $\mathrm{z}$-axis to fluctuate periodically due to cosine and sine functions in the matrix. This however is not the case for the y-axis because it is the rotation axis, which does not have the cosine and sine functions. If the $\mathrm{x}$ - and $\mathrm{z}$-axis errors are constantly positive or negative over the whole rotation, the errors will then reduce to zero after the whole rotation period Therefore, multiplication of $\delta \omega_{i b}^{b}, \delta f b$ and $C_{b}^{n}$ in the equations affects the INS navigation accuracy. The idea of the proposed approach therefore lies in the periodical change (modulation) of the elements in $C_{b}^{n}$ such that the average of $C_{b}^{n} \delta \omega_{i b}^{b}$ and $C_{b}^{n} \delta f^{b}$ approximate to zero. This approach is therefore may eliminate the constant error terms on IMU axes that are perpendicular with the rotation axis.

\subsection{Observability of error}

Observability is defined as the ability to determine estimation from a given sequence of measurements. The principle of the rotating IMU in improving the error states observability can be explained by assuming a simple case of a stationary and level IMU. Because heading drift is closely related with attitude error on $\mathrm{z}$ axis of an IMU, therefore remaining discussion on this section will show only the attitude error example.

For attitude errors, the North and East attitude errors are observable through the velocity error updates because there is a large force in the Down axis resulting from the gravity heading when stationary (Hide, 2003). Therefore we would like to see how the rotation effect makes the attitude error in the Down axis more observable, where the error is not observable for the normal IMU when stationary.
The attitude error model can be rewritten as (ignoring other terms because of the low cost IMU used) (Godha and Lachapelle, 2008) Equation 3:

$\dot{\epsilon}=-C_{b}^{n} \delta \omega_{i b}^{b}$

When stationary and level, (3) becomes Equation 4:

$$
\left(\begin{array}{l}
\dot{\epsilon}_{N} \\
\dot{\epsilon}_{E} \\
\dot{\epsilon}_{D}
\end{array}\right)=\left(\begin{array}{lll}
1 & 0 & 0 \\
0 & 1 & 0 \\
0 & 0 & 0
\end{array}\right)\left(\begin{array}{c}
\delta \omega_{x} \\
\delta \omega_{y} \\
\delta \omega_{z}
\end{array}\right)
$$

When the IMU rotates 900 about its $y$-axis, the $C_{b}^{n}$ changes and (4) becomes:

$$
\left(\begin{array}{l}
\dot{\epsilon}_{N} \\
\dot{\epsilon}_{E} \\
\dot{\epsilon}_{D}
\end{array}\right)=-\left(\begin{array}{ccc}
0 & 0 & -1 \\
0 & 1 & 0 \\
1 & 0 & 0
\end{array}\right)\left(\begin{array}{l}
\delta \omega_{x} \\
\delta \omega_{y} \\
\delta \omega_{z}
\end{array}\right)
$$

Equation 5 shows that the gyro sensor error on the zaxis is now made observable through the North attitude error. Because the North attitude errors are already observable from velocity updates, the correlated gyro sensor error on z-axis (i.e., z-axis gyro bias) can therefore be observed as well.

This simple example assumes a simple case of the IMU when it is stationary and level. However it does explain how by simply rotating the IMU one can improve the observability of the error states. In practical, however, many terms during the modelling and estimation process may contain errors. For example, $C_{b}^{n}$ and $\left(f^{n} \times\right)$ may contain errors and the state transition matrix may not correctly model the propagation of error. Nonetheless by also improving the observability of the error states, more information is updated to the estimation filter such as Kalman Filter (KF). During the estimation process, these errors may correlate with the other error states and over time, the KF can propagate more information about the uncertainty of all the error states. This will therefore provide better error estimation of the system.

\section{REAL WALKING TRIALS}

\subsection{Trial Description}

The field trial was performed using the rotatedIMU prototype, shown in Fig. 1. The IMU used was 
from MicroStrain (3DM-GX3-25). It should be a reasonable representation of a low cost sensor, with typical technical specifications of a low cost IMU grade with a dimension of $44 \times 25 \times 11 \mathrm{~mm}$ and weighing only $11.5 \mathrm{~g}$. The accelerometer bias stability is quoted as $\pm 0.01 \mathrm{~g}$ and for the $300 \% \mathrm{~s}$ model, the gyro biases are specified as $\pm 0.2 \%$ s. The particular IMU used has a limit of $1200 \%$ for angular rotation and $18 \mathrm{~g}$ for acceleration. The exact rotation rate for the IMU platform is unknown.

The rotating of the IMU platform was started from the beginning (when powering the device). A user equipped with the RIMU stood on the starting position so that the IMU horizontal alignment could be made for approximately $1 \mathrm{~s}$ at the beginning of the walk. He then performed two walks (back to back) around the office area. This created a rectangular trajectory around the office, where the start and the end trajectory is the same, marked by a tape.

The first walk with the RIMU was for 10 rounds. At the end of the 10th round, the RIMU mode was turned off by stopping the platform rotation using the switch, whilst keeping the IMU switched on. The user walked again immediately for the second walk with another 10 rounds on approximately the same trajectory (by following straight features on the floor carpet). The two trials lasted for about $650 \mathrm{~s}$ each. The reference for comparison of error estimation was created based on the second walk, in which a method developed by authors (Abdulrahim et al., 2010; 2011) was applied. The example of raw acceleration data from the IMU is plotted in Fig. 2 to show the RIMU in operation. Fig. 3 shows an example of the detected ZUPT events for the RIMU field trial. The data was processed and analyzed in a Kalman Filter (KF) environment using POINT software (Hide, 2009).

\subsection{Rotation Rate}

A simulated test was first established to check the outcome of having different rates for platform rotation, where the IMU was to be mounted. This is shown in Fig. 4, in terms of the Time-To-Converge (TTC), for the z-axis gyro bias during stationary condition.

Fig. 4 shows the different rotation rates used, which were $0.6,1$ and $6 \%$, when the IMU was rotated continually. It is observed from the figure that a faster rotation rate resulted in a quicker convergence to the true simulated bias. Using the rotation rate of 6 $\%$ resulted in the z-axis gyro bias being resolved to within $0.1 \%$ after approximately $100 \mathrm{~s}$. Conversely, using slower rotation rates of 1 and $0.6 \%$ resulted in the $\mathrm{z}$-axis gyro bias being resolved to within $0.1 \%$ only after approximately 250 and $750 \mathrm{~s}$ respectively, approximately slower by 150 and $650 \mathrm{~s}$ from the former case. It is, however, not considered a big issue to select which is the best rotation rate since the main goal of the approach was to show how the rotating IMU can be an advantage to mitigate heading drift error and not to compare how quick the convergence rate for the estimation of errors because position solution is the parameter of concern, not the rotation rate.

\subsection{Rotation Scheme}

After the rotation rate had been chosen, the different types of RIMU schemes needed to be identified. The RIMU scheme is defined as to how the rotation is performed for the RIMU. This was done to see if there was any benefit in utilizing different schemes and if it was, whether it was feasible practically.

Therefore, five different schemes of rotation about the $y$-axis have been tested with the chosen platform rotation rate of $6 \%$ from previous section. The schemes were named as schemes 1,2, 3, 4 and 5; where scheme 1 $=$ continuous IMU rotation; scheme $2=360^{\circ}$ rotation back and forth; scheme $3=180^{\circ}$ rotation back and forth; scheme $4=90^{\circ}$ rotation back and forth and scheme $5=$ $45^{0}$ rotation back and forth. The result of using different schemes during the stationary condition is depicted in Fig. 5 in terms of Time-To-Converge (TTC) for the $\mathrm{z}$ axis gyro bias estimates.

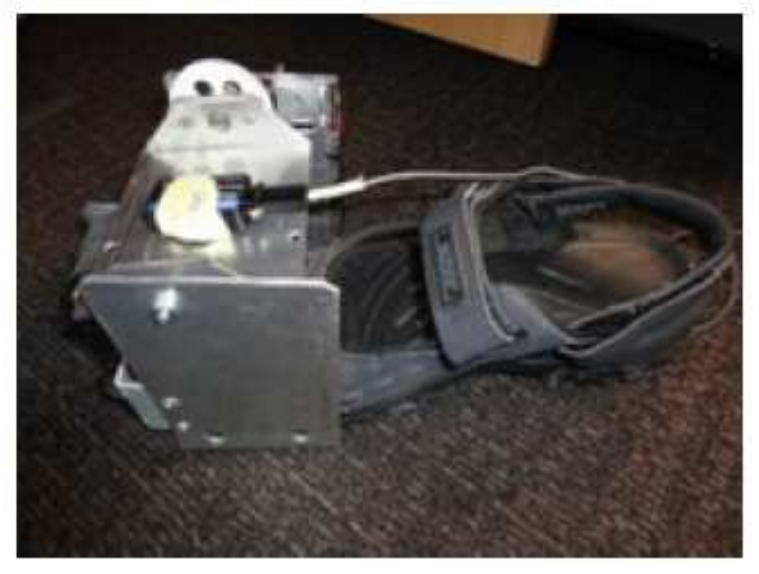

Fig. 1. RIMU prototype with IMU mounted on a rotating platform 


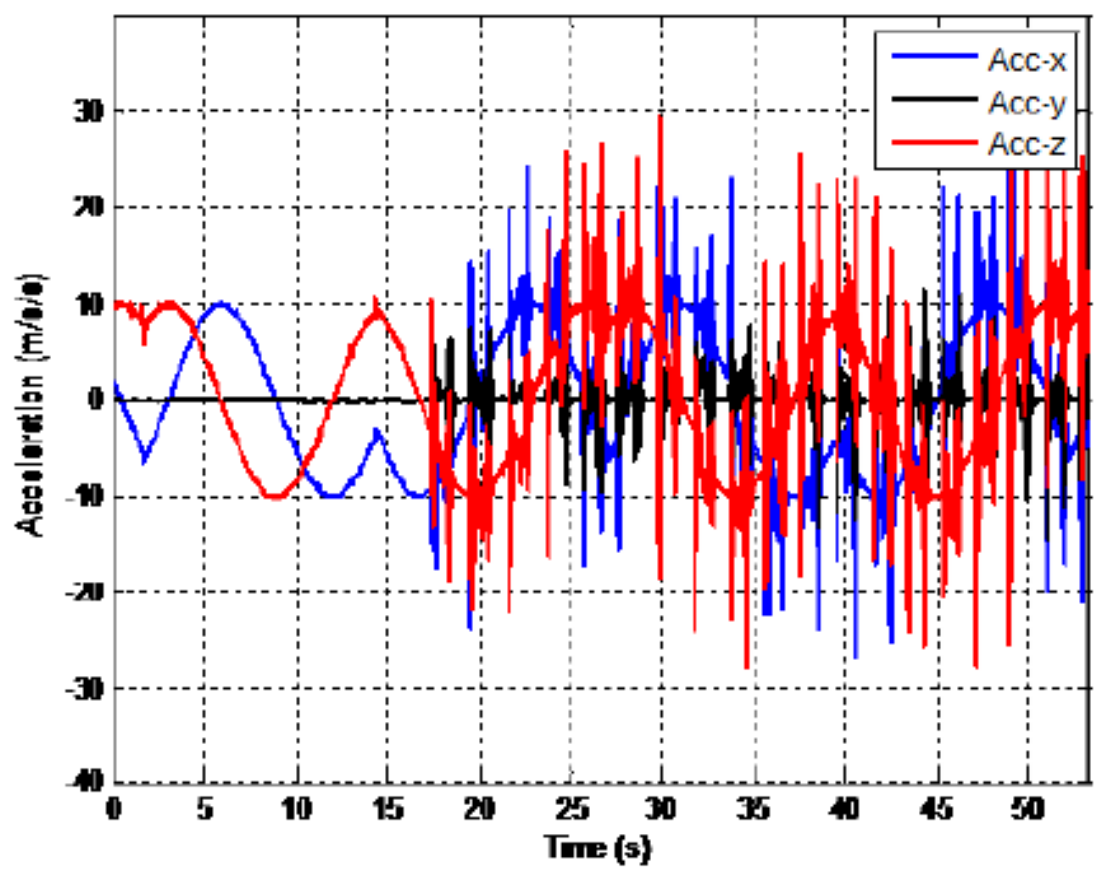

Fig. 2. The actual raw IMU acceleration data

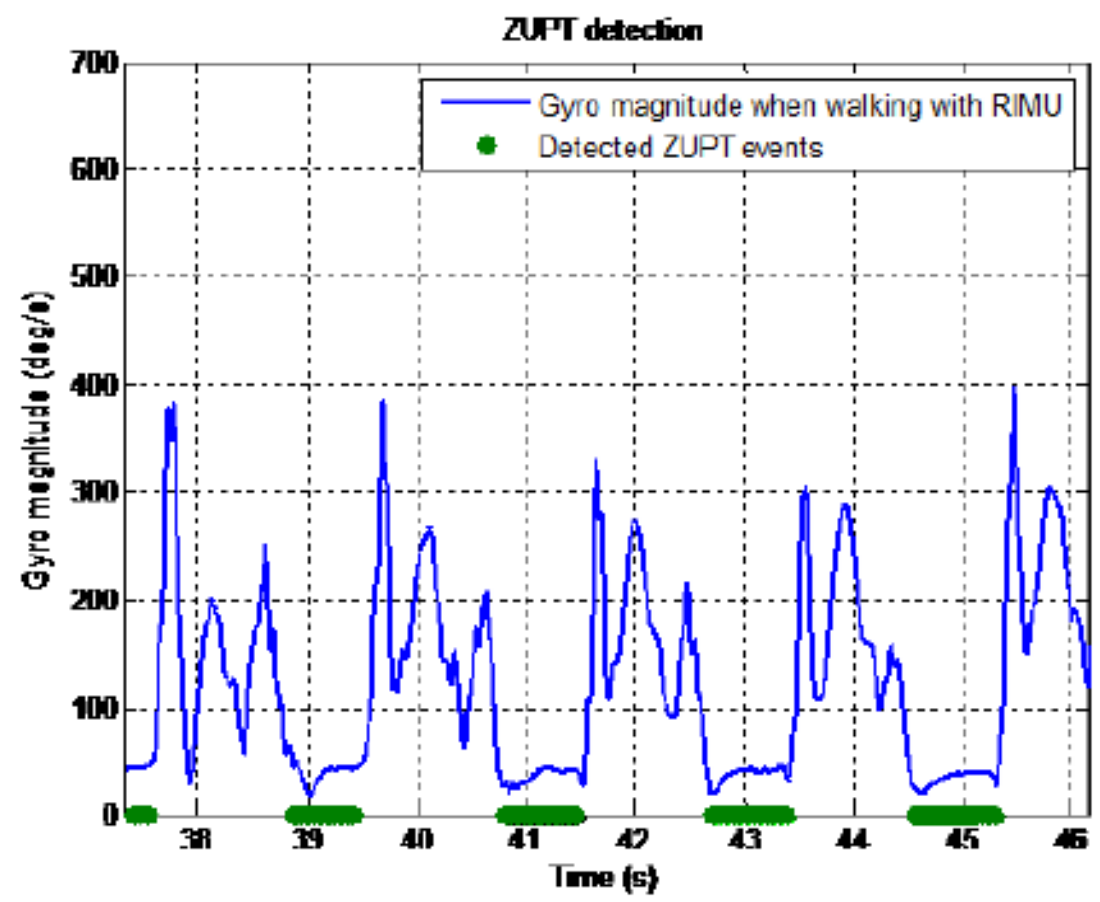

Fig. 3. Example of ZUPT detection for the trial 


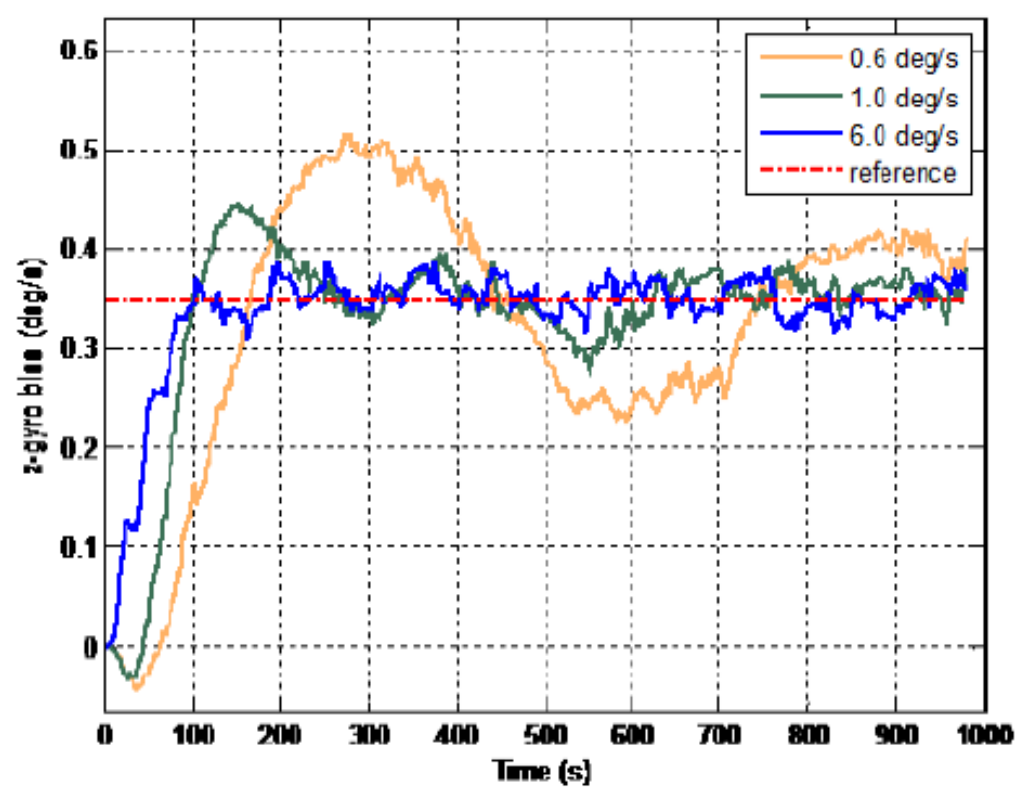

Fig. 4. Comparison of z-axis gyro bias when different rotation rates are used

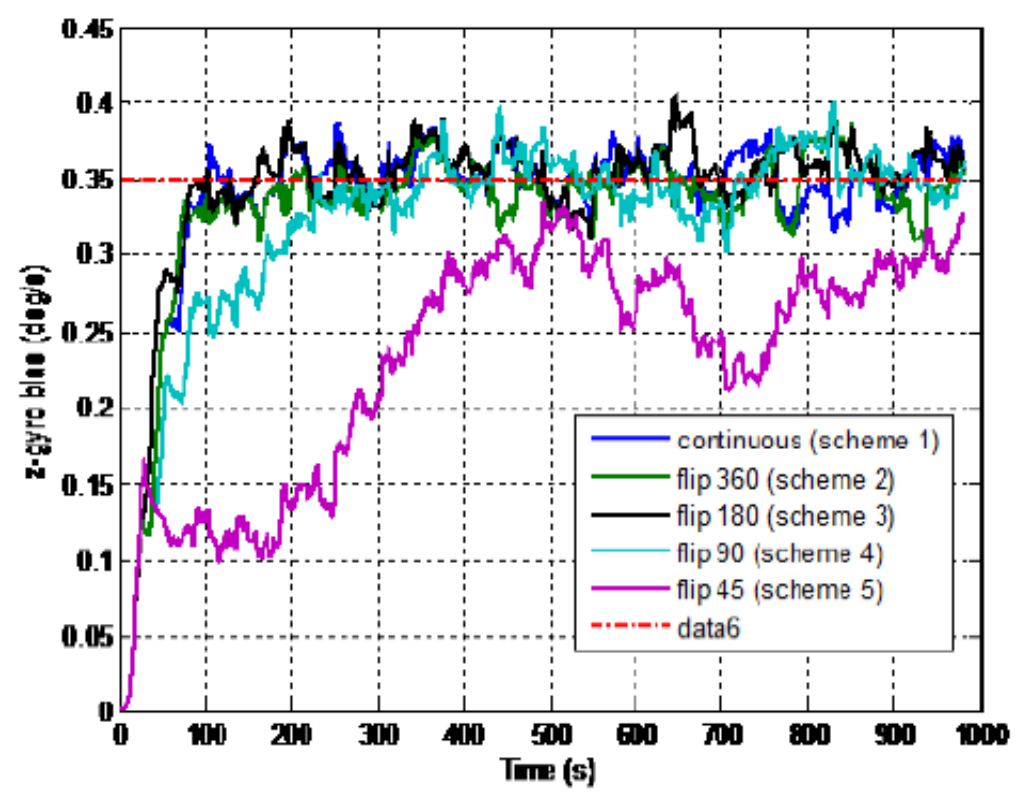

Fig. 5. Z-axis gyro bias when different rotation schemes were used

From Fig. 5, it is observed that all schemes converged to the reference. Nevertheless, when the flipping effect of the IMU changed from scheme 1 (continuous rotation) to scheme 5 (450 rotation back and forth), the TTC increased from approximately $100 \mathrm{~s}$ to 1000 s. Scheme 1, 2 and 3 have a similar convergence rate, resolving to within $0.01 \%$ s after approximately $100 \mathrm{~s}$, while schemes 4 and 5 converged slower at approximately 300 and 1000 s respectively.

Although schemes 1, 2 and 3 showed similarity, scheme 2 was chosen for the rotating IMU because it was more practical when the rotating IMU platform was 
mounted on a shoe for the field trial. This was in order not to tangle the IMU data cables so much. If scheme 1 is selected, the data cable from the IMU to the data logger might become tangled. Nevertheless, it might be possible to use schemes 1 and 3 for future work if the IMU has the capability to operate wirelessly. Furthermore, although smaller rotation (schemes 4 and 5) may be practically easier to perform without tangling the cables, it was not chosen because of the slow convergence rate.

\section{RESULTS}

For the modelled IMU errors in this trial, which are the accelerometer bias error and gyro bias error, only zaxis gyro bias is presented because it is considered the unobservable state when IMU is used with ZUPT only. As discussed before, this error is thought to be the main contributor causing heading drift. Fig. 6 shows the z-axis gyro bias for rotating IMU and non-rotating IMU, plotted against the reference. It is the estimates obtained from the Kalman filter used. Both datasets were initialised with its average bias values during alignment.

It can be observed from the figure that the rotating IMU has a similar plot to the reference, as opposed to non-rotating IMU. After about 50s, the rotating IMU has resolved and stabilized to within $0.050 / \mathrm{s}$ from the reference, whilst the non-rotating IMU has not yet resolved to the reference, even until the end of the trial. This indicates that $\mathrm{z}$-axis gyro bias is made observable through the use of the rotating IMU as opposed to the non-rotating IMU where the z-axis gyro bias converged to a wrong value. Note that the slight difference of results between the rotating IMU and the reference is due to the difference in time when the two trials were performed.

Next, the rotating IMU benefit in mitigating heading drift to the overall low-cost IMU performance is assessed using its position solution. Statistical comparison for the position solution in this section is tabulated in Table 1 using four criteria: Return Position Error (RPE) in meters, percentage of RPE from the total distance travelled, mean of Horizontal Position Error (HPE) and maximum Position Error (PE) both in meters. Fig. 7 shows an example of position solution for Trial \#2.

From Trial \#2, when the rotating IMU was not used, the maximum PE for North and East is 10.7 and $7.0 \mathrm{~m}$ respectively. In contrast, implementing the rotating IMU has reduced the North PE by more than tenfold, where the maximum PE falls to $1.3 \mathrm{~m}$. Likewise, for East PE, implementing RIMU reduces the East PE by about the same amount, with maximum East PE now only $0.8 \mathrm{~m}$. The mean HPE is computed by taking an average of the HPE, where HPE is represented by the square root of the sum of the squared of North and East PEs. For this trial, the mean HPE was computed to be only $0.60 \mathrm{~m}$ when rotating IMU was implemented, but increases to $2.68 \mathrm{~m}$ when rotating IMU was not implemented.

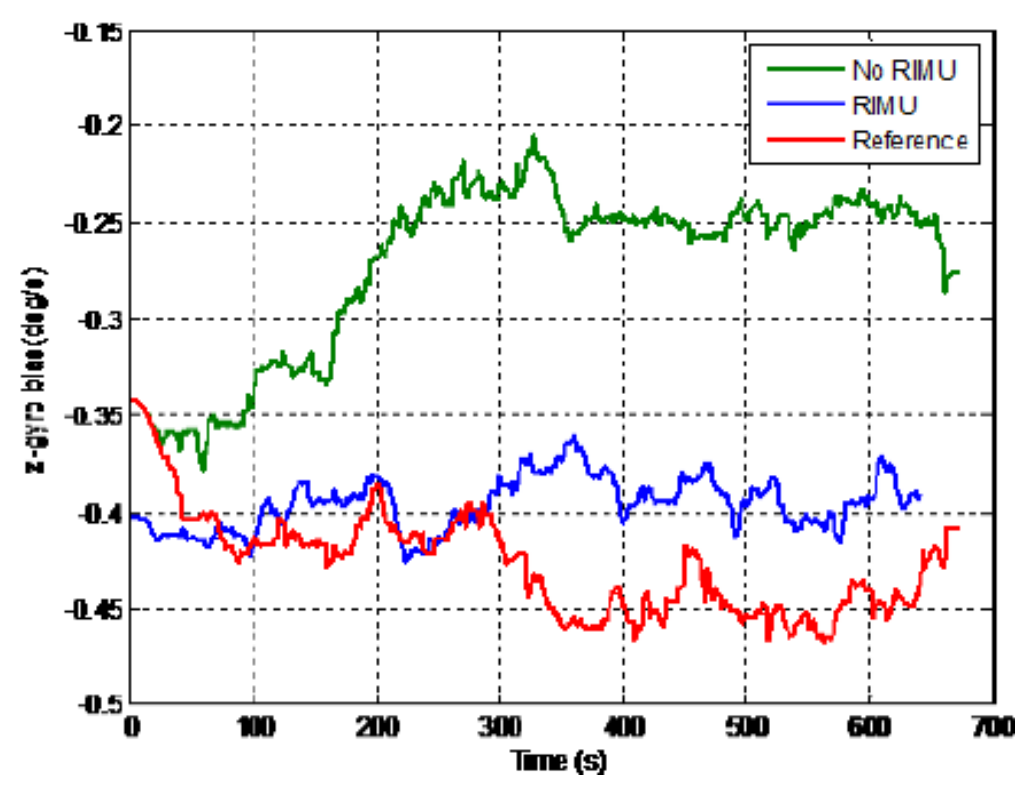

Fig. 6. Estimation of z-axis gyro bias with different approaches 

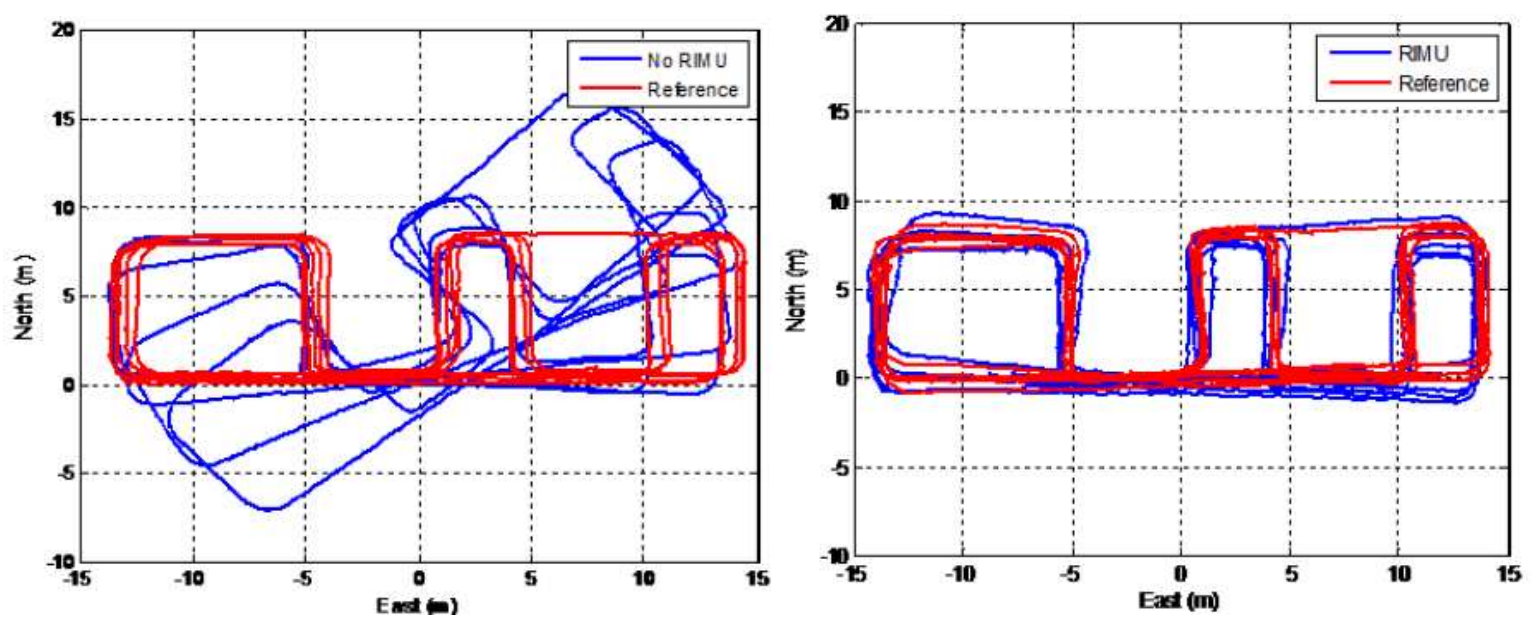

Fig. 7. Position solution for non-rotating IMU (left) and the proposed rotating IMU (right)

Table 1. Position solution comparison between rotating IMU and non-rotating IMU

\begin{tabular}{|c|c|c|c|c|c|c|c|}
\hline \multirow[b]{2}{*}{ Trial } & \multirow[b]{2}{*}{ Method } & \multirow[b]{2}{*}{$\begin{array}{l}\text { Duration } \\
\text { (min) }\end{array}$} & \multirow[b]{2}{*}{$\begin{array}{l}\text { Total } \\
\text { dist. (m) }\end{array}$} & \multicolumn{2}{|c|}{ Max. PE (m) } & \multirow[b]{2}{*}{$\begin{array}{l}\text { RPE } \\
(\mathrm{m})\end{array}$} & \multirow[b]{2}{*}{$\begin{array}{l}\text { Mean } \\
\text { HPE (m) }\end{array}$} \\
\hline & & & & North & East & & \\
\hline \multirow[t]{2}{*}{1} & No-RIMU & 11.2 & 385.3 & 10.9 & 12.42 & 12.35 & 4.59 \\
\hline & RIMU & 10.7 & 354.0 & 0.6 & 0.70 & 0.80 & 0.42 \\
\hline \multirow[t]{2}{*}{2} & No-RIMU & 14.3 & 475.4 & 10.7 & 7.00 & 3.36 & 2.68 \\
\hline & RIMU & 13.9 & 475.7 & 1.3 & 0.80 & 0.15 & 0.60 \\
\hline \multirow[t]{2}{*}{3} & No-RIMU & 15.0 & 548.8 & 6.8 & 8.00 & 6.79 & 3.14 \\
\hline & RIMU & 15.5 & 545.2 & 2.9 & 1.80 & 1.27 & 1.93 \\
\hline \multirow{2}{*}{ MEAN } & No-RIMU & 13.5 & 469.8 & 9.5 & 9.10 & 7.50 & 3.50 \\
\hline & RIMU & 13.4 & 458.3 & 1.6 & 1.10 & 0.74 & 1.00 \\
\hline
\end{tabular}

Two more real walking trials were performed on a different trajectory to check whether the positioning accuracy result achieved previously can be repeated. Table 1 summarizes the performance of using the rotating IMU results from the three indoor trials.

For the rotating IMU, the three trials lasted for an average duration of $13.4 \mathrm{~min}$. The average distance travelled is $458.3 \mathrm{~m}$. From these three rotating IMU trials, an average maximum Position Error (PE) for North and East were computed to be only 1.6 and $1.1 \mathrm{~m}$ respectively. The average RPE was also relatively better at only $0.74 \mathrm{~m}$, or only below $0.2 \%$ from the total distance travelled.

For the non- rotating IMU, the three trials lasted for an average duration of $13.5 \mathrm{~min}$ with an average distance of $469.8 \mathrm{~m}$. In contrast with the rotating IMU case, an average maximum PE for North and East when the RIMU was not implemented are relatively higher at 9.5 and $9.1 \mathrm{~m}$ respectively. Additionally, the average RPE also increases by tenfold to $7.5 \mathrm{~m}$, or above $1 \%$ from the total distance travelled. Furthermore, the maximum rotating IMU Horizontal Position Error (HPE) computed from North and East maximum PE, on average was found to be only $1.9 \mathrm{~m}$ whilst for the non-rotating IMU, the maximum HPE was relatively worst at $13.2 \mathrm{~m}$. This, therefore, indicates a significant improvement in position by reducing more than $85 \%$ of maximum HPE when the method of rotating the IMU was implemented.

Although the results look promising, one issue that needs to be highlighted is the impact of heading initialization issue to the overall position solution. We do know that the heading would be drifting if the IMU was not rotating. Similarly, this will affect the rotating IMU as well. When rotating is performed, the IMU will still take some time before it can observe the error. Therefore, before we can fully observe the $\mathrm{z}$ axis gyro bias (assuming that the modelled gyro bias is the only error affecting the gyro z-axis measurement), the heading will then still be drifting. 
This will eventually impact the whole position solution because the initial heading (at the start of the walk) will be wrong.

Therefore, an issue to consider when using the RIMU technique is the initialization of the heading for the 'lowest-cost' IMU (or uncalibrated IMU). Standard coarse alignment for a strap down IMU would be to set the IMU initial heading during coarse alignment, based on information from for example the GPS heading or magnetometer. The same approach however cannot be applied to the RIMU.

Consider this. Suppose the IMU horizontal alignment is performed for $1 \mathrm{~s}$ (and the heading is initialized manually) and the walk is performed after 20 s. Suppose also the true initial heading is $45^{\circ}$ and the low-cost RIMU drifts at a rate of $10 \%$. If the RIMU heading is manually set during heading alignment to $45^{\circ}$, the actual initial heading at the start of the walk (at $20 \mathrm{~s}$ ) for the RIMU would be different. This is because the RIMU heading will still drift because the z-axis gyro bias will take some time to be observed by the RIMU. After $20 \mathrm{~s}$, the IMU heading would have been $200^{\circ}$ off from the true heading. This will cause the actual initial heading (used for the IMU mechanization) to be $245^{\circ}$, which is wrong. This subsequently will affect the position computation afterwards (although the heading drift after this period would be reduced because of the RIMU effect).

Nevertheless, this case assumed that the $\mathrm{z}$-axis gyro bias was zeroed during initialization. Usually in practice, gyro biases were initialized with its average values taken during stationary alignment, where the values might have been similar to the actual values. This therefore gives some information to the KF when estimating the biases and the heading errors. The only probable dilemma is if the initialized gyro bias value (using its average values during alignment) may not represent the correct estimation of the true values (the uncertainty is too large). For example, the biases might change so much and very rapidly, subsequently affecting the estimation of the heading error even when initialized properly. If this is the case, then the heading initialization issue must be addressed appropriately.

\section{CONCLUSION}

The concept of a rotating IMU was investigated by presenting real walking trials. An approach of a rotating IMU on a $y$-axis for the low-cost foot-mounted pedestrian navigation was first presented. It was demonstrated using real field trials that it is able to mitigate heading drift error resulting in a reduced position drift error without the need to have any other external measurements apart from available ZUPTs.

An investigation was first made on the observability of the $z$-axis gyro bias (which corrupted the IMU heading) that was unobservable in a system that had no other external measurement update except ZUPTs. Real field trials were performed using a prototype of a rotating low-cost foot-mounted IMU. The results were analysed in terms of IMU error observability and the position solution accuracy.

Using the rotating approach, it was shown in the real walking trial that IMU z-axis gyro error observability increased. As a result of improved error observability resulting from the rotating IMU, the heading drift was mitigated. This was clearly shown in the comparison of position solution where on an average of $13 \mathrm{~min}$ of walking, the average of maximum HPE was below $2 \mathrm{~m}$ when the RIMU was implemented. In contrast, without implementing the RIMU, the maximum HPE was more than $9 \mathrm{~m}$.

Different rotation rates and schemes were also presented. Although at the moment only a proof of concept is available, it is not impossible to implement considering a promising result presented herein with a very minimal modification to an existing IMU footmounted pedestrian navigation system.

\section{ACKNOWLEDGMENT}

Authors would like to thank Ministry of Education of Malaysia and Universiti Sains Islam Malaysia (USIM) for partly sponsoring the study.

\subsection{Author's Contributions}

Khairi Abdulrahim: Performed all experiments, comprehensively analyzed the data and the main/corresponding author of the manuscript.

Chris Hide: Participated in all experiments, coordinated the data-analysis and contributed to the writing of the manuscript.

Terry Moore: Organized the study, contributed in the data-analysis and to the writing of the manuscript.

Chris Hill: Organized the study, contributed in the data-analysis and to the writing of the manuscript.

\subsection{Ethics}

No ethical issues, to the authors' knowledge, may arise as a result of the publication of this article. 


\section{REFERENCES}

Abdulrahim, K., C. Hide, T. Moore and C. Hill, 2010. Aiding MEMS IMU with building heading for indoor pedestrian navigation. Proceedings of the Ubiquitous Positioning Indoor Navigation and Location Based Service, Helsinki, Finland, Oct. 14-15, IEEE Xplore Press, Kirkkonummi, pp: 1-6. DOI: $10.1109 /$ UPINLBS.2010.5653986

Abdulrahim, K., C. Hide, T. Moore and C. Hill, 2011. Aiding low cost inertial navigation with building heading for pedestrian navigation. J. Navigat., 64: 219-233. DOI: 10.1017/S0373463310000573

Alvarez, J., D. Alvarez, A. Lopez and R.C. Gonzalez, 2012. Pedestrian navigation based on a waistworn inertial sensor. Sensors, 12: 10536-10549. DOI: $10.3390 / \mathrm{s} 120810536$

Foxlin, E., 2005. Pedestrian tracking with shoe-mounted inertial sensors. IEEE Comput. Graph. Applic., 25: 38-46. DOI: 10.1109/MCG.2005.140
Godha, S. and G. Lachapelle, 2008. Foot mounted inertial system for pedestrian navigation. Measure. Sci. Technol., 19: 075202-075202.

DOI: 10.1088/0957-0233/19/7/075202

Hide, C., 2003. Integration of GPS and low cost INS measurements. Ph.D. Thesis, University of Nottingham.

Hide, C., 2009. Algorithm documentation for POINT software. Geospatial Research Centre, New Zealand.

Jiménez, A.R., F. Seco, F. Zampella, J.C. Prieto and J. Guevara, 2012. Improved heuristic drift elimination with magnetically-aided dominant directions (MiHDE) for pedestrian navigation in complex buildings. J. Locat. Based Services, 6: 186-210. DOI: 10.1080/17489725.2012.687779

Klein, I., S. Filin, T. Toledo and I. Rusnak, 2012. Assessment of aided-INS performance. J. Navigat., 65: 169-185.

DOI: $10.1017 / \mathrm{S} 0373463311000609$ 